\title{
The importance of timely introduction of vancomycin therapy against methicillin-resistant Staphylococcus aureus (MRSA) bacteremia and severity of MRSA bacteremia at Teaching Hospital, Anuradhapura, Sri Lanka
}

\author{
Jayaweera Arachchige Asela Sampath Jayaweera ${ }^{1}$, Malika Karunarathne ${ }^{2}$ and Wikum Widuranga Kumbukgolla ${ }^{3}$
}

1. Department of Microbiology, Faculty of Medicine and Allied Sciences, Rajarata University Saliyapura, Sri Lanka;

2. Consultant Microbiologist, Teaching Hospital, Anuradhapura, Sri Lanka; 3. Department of Biochemistry, Faculty of Medicine and Allied Sciences, Rajarata University Saliyapura, Sri Lanka.

Corresponding author: Jayaweera Arachchige Asela Sampath Jayaweera, e-mail: jaas820703@yahoo.com,

Co-authors: MK: gamagemk@gmail.com,WWK: kumbukgolla@yahoo.com

Received: 01-03-2017, Accepted: 31-03-2017, Published online: 25-04-2017

doi: 10.14202/IJOH.2017.7-11 How to cite this article: Sampath Jayaweera JAA, Karunarathne M, Kumbukgolla WW. The importance of timely introduction of vancomycin therapy against methicillin-resistant Staphylococcus aureus (MRSA) bacteremia and severity of MRSA bacteremia at Teaching Hospital, Anuradhapura, Sri Lanka. Int J One Health 2017;3:7-11.

\begin{abstract}
Aim: Worldwide, an estimated 2 billion healthy people carry Staphylococcus aureus (SA) and of these, up to 53 million are thought to carry methicillin-resistant SA (MRSA). MRSA bacteremia patients are more critical to manage and timely introduction of antibiotics is life-saving. The aim of the study was to elucidate the prevalence of MRSA bacteremia in different units of Teaching Hospital, Anuradhapura (THA), Sri Lanka and assess the clinical characteristics and associated mortality related to timely introduction of vancomycin therapy.
\end{abstract}

Materials and Methods: The data on MRSA bacteremia which were obtained from THA, for the period of March 2012 to December 2013 were statically analyzed emphasizing the unit-wise prevalence, severity, and comorbidity and timely introduction of vancomycin therapy.

Results: The laboratory records of total 13,260 blood cultures were analyzed. Of those, MRSAbacteremia was detected in 61 cultures $(9.3 \%)$. The highest prevalence of MRSA bacteremia was observed in the nephrology unit. The survival rate of the patients when the vancomycin therapy started before $24 \mathrm{~h}$ of receiving the blood culture report was $94.9 \%$ and in the instances of the treatment started after $24 \mathrm{~h}$ of blood culture report, the survival rate decreased down to $50 \%$. High Pitt Bacteraemia score (PBS) (p<0.05) and initiation of vancomycin therapy after $24 \mathrm{~h}$ following the receipt of blood culture report $(\mathrm{p}<0.05)$ independently affected the MRSA bacteremic patient's $7^{\text {th }}$ day mortality. Having comorbidities have not shown significant impact on $7^{\text {th }}$ day mortality.

Conclusion: The start of vancomycin therapy as earlier as possible following arrival of antibacterial susceptibility test reduces the likelihood of mortality.

Keywords: MRSA bacteremia, timing of vancomycin, severity.

\section{Introduction}

Worldwide, an estimated two billion healthy people carry Staphylococcus aureus (SA) and of these, up to 53 million are thought to carry methicillin-resistant SA (MRSA). SA is a feared pathogen because it causes severe infections and spread by metastatic foci. SA commonly colonizes the nostrils, skin, perineum, respiratory tract, open wounds, and urinary tract which serves potential sites for infection $[1,2]$. The multidrug-resistant strains of SA have been isolated. In general, the fatality rates of MRSA infections range from $20 \%$ to $50 \%[2,3]$.

Over the past decades, the incidence of SA bacteremia in hospitals and in community has significantly increased [1]. SA has become the leading cause of

Copyright: Sampath Jayaweera, et al. This article is an open access article distributed under the terms of the Creative Commons Attribution 4.0 International License (http://creativecommons.org/ licenses/ by/4.0/), which permits unrestricted use, distribution, and reproduction in any medium, provided you give appropriate credit to the original author(s) and the source, provide a link to the Creative Commons license, and indicate if changes were made. The Creative Commons Public Domain Dedication waiver (http:// creativecommons.org/ publicdomain/zero/1.0/) applies to the data made available in this article, unless otherwise stated. hospital-acquired bacteremia, particularly in the intensive care units (ICUs) where nosocomial bacteremia is one of the leading causes of death. With emergence of MRSA, it has received even more attention because MRSA bacteremia causes high morbidity and mortality. Over the past 3 decades, numerous outbreaks of MRSA bacteremia have been reported in hospitals in South Asia including Sri Lanka [4]. The therapeutic options are limited to the use of glycopeptide and linezolid in treating MRSA bacteremia $[2,5,6]$.

This study was conducted to assess the therapeutic outcome of vancomycin treatment depending on the time-point of its introduction after obtaining blood culture reports. Unit-wise prevalence of MRSA at Teaching Hospital, Anuradhapura (THA), severity and comorbidity related to MRSA bacteremia was analyzed to indicate possible modes of transmission and reservoirs.

\section{Materials and Methods}

\section{Ethical approval}

The ethical clearance for this study was obtained from Faculty of Medicine and Allied Sciences, Rajarata University of Sri Lanka. 


\section{Sample collection and isolation of MRSA from posi- tive blood cultures}

This work was carried out at the THA, Sri Lanka. THA is an approximately 1839 bed tertiary care medical facility consisting of a University Professorial Teaching Unit; located in Anuradhapura, North Central Province of Sri Lanka. The hospital serves to a population of 1.2 million in the North Central province having 106,032 admissions in 2011, and in 2012, the hospital received 110,021 admissions [7]. Here, we analyzed the microbiology laboratory records and bed head tickets (BHT) of SA and MRSA bacteremia patients admitted to THA from March 2012 to December 2013. All the clinical investigations have been done by a consultant physician and microbiological investigations have been conducted under the direct observation of the consultant microbiologist at THA.

Microbiological investigations have been conducted using the BacTec ${ }^{\circledR}$ 3D automated blood culture machine and the disc diffusion test has been performed according to MRSA susceptibility panel of antimicrobials including vancomycin susceptibility [8]. Cases of culture-proven SA infection with bacteremia were enrolled in the study using the information obtained from BHT and laboratory records. Admission books and record room data were used to locate the unit of patient's admission. Mortality was calculated according to postmortem reports.

\section{MRSA bacteremia prevalence and timing of anti- MRSA therapy}

The prevalence of MRSA bacteremia rates was calculated in different units including ICUs. Since vancomycin is prescribed as a main anti-MRSA treatment timing between vancomycin susceptibility results and initiation of vancomycin therapy was calculated.

\section{Assessment of severity and outcome following MRSA bacteremia}

Severity of bacteremia was assessed using Pitt Bacteraemia score (PBS) [9], and comorbidity was evaluated using Charlson comorbidity (CC) index [10]. Clinical evolution was classified as a cure when the clinical manifestations disappeared and the cultures became negative and as failure when there was persistence of the clinical manifestations, persistence of positive cultures, or evidence of death due to the MRSA bacteremia. Death was considered to be due to the MRSA bacteremia when at least 1 of the following criteria was satisfied: A culture positive for MRSA from a sample obtained at the time of death (hemoculture), persistent septicemic symptoms, or occurrence of death without any explanation within the first 7 days after the diagnosis of MRSA bacteremia. Deaths that did not satisfy one of these criteria were considered to be a consequence of the patient's underlying disease. The follow-up period was from the diagnosis of bacteremia (the point where the positive results for MRSA on hemoculture) until cure or death.

\section{Statistical analysis}

The data were double checked and transported to SAS 9.1 (2005 New Jersey, USA) for statistical analysis [11]. Demographic data were in measures of central tendency. The Chi-squared test was performed to assess the factors associated with survival and mortality following after $7^{\text {th }}$ day of MRSA bacteremia.

\section{Results}

A total sample of 13,260 blood cultures were investigated for possible bacteremia obtained from the patients admitted to THA. Of those, 1352 blood cultures were identified as bacteremia and the presence of SA bacteremia was detected in 655. Out of 655 cultures, 61 (9.3\%) indicated the presence of MRSA. It was further calculated that $0.28 \mathrm{MRSA}$ bacteremia incidents per 1000 admissions.

The highest prevalence $(52.4 \%)$ of MRSA bacteremia was observed among the people who aged $>60$ years (Figure-1).

Furthermore, it was detected more commonly in males $(67.3 \%)$ than the females $(32.7 \%)$. Nephrology unit, orthopedic unit, and ICUs hosted comparatively higher MRSA bacteremia cases accounting for $15(24.6 \%), 14(23 \%)$, and $13(21.3 \%)$, respectively (Figure-2). The average period of hospitalization of a MRSA bacteremia patient was $26.2 \pm 6.4$ days.

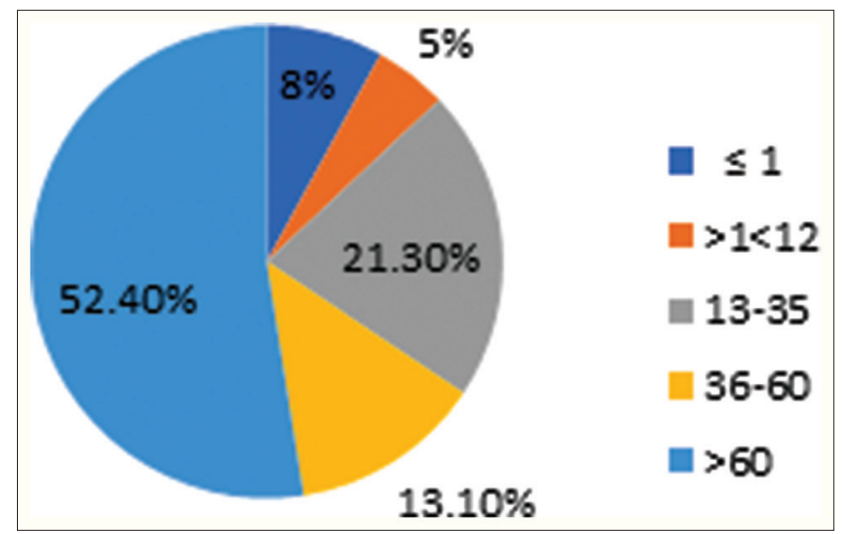

Figure-1: Age-wise distribution of methicillin-resistant Staphylococcus aureus bacteremia.

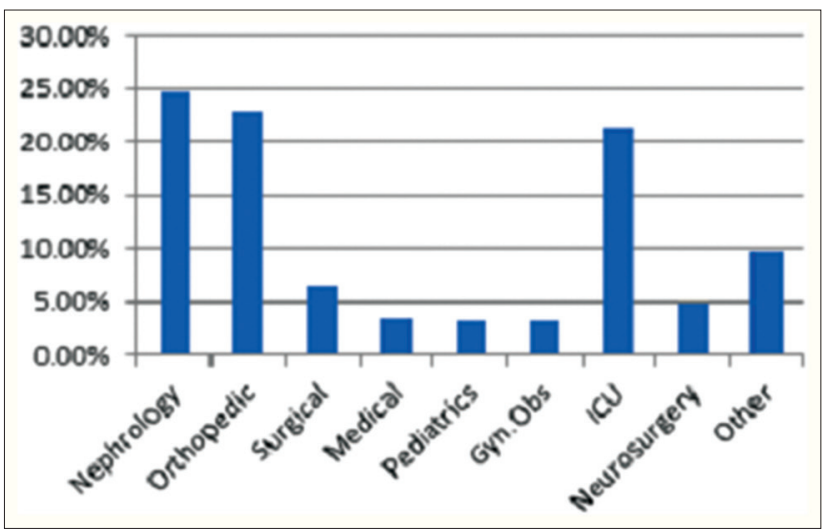

Figure-2: Prevalence of methicillin-resistant Staphylococcus aureus bacteremia according to unit of patient's admission. 
When the risk factors for acquisition of MRSA bacteremia is considered; $26(40 \%)$ were with peripheral cannula; $16(42.6 \%)$ were on peritoneal dialysis; $16(26.2 \%)$ were with a urinary catheter; $4(6.5 \%)$ were with A-V shunts; 6 (9.8\%) were with intubation. Furthermore, $10(16.4 \%)$ were undergone previous surgeries and $5(8.2 \%)$ were following chemotherapy. $10(16.4 \%)$ were associated with polytrauma. With respect to comorbidities in MRSA bacteremia, the CC index was $4.8 \pm 1.3$ and the severity of MRSA infection, PITTS score, was $8.3 \pm 1.5$. Fever $(80.8 \%)$ and local signs $(78.8 \%)$ were the most common clinical manifestations.

At the time of receiving the positive results of blood cultures, 49 were on third-generation cephalosporin; 5 were on cloxacillin, and another 5 were on co-amoxiclav. Only 2 patients had been kept without antibiotics. After detecting MRSA in blood culture, the antibiotic administered was changed to glycopeptide in all the patients.

39 patients were changed into vancomycin within $24 \mathrm{~h}$ of obtaining the results (Group 1). Further 10 patients were changed into vancomycin after $24 \mathrm{~h}$ of receiving blood culture results (Group 2). Seven patients were died within the $1^{\text {st }}$ week $\left(7^{\text {th }}\right.$ day mortality) of positive blood culture report as a consequence of septicemia (Table-1).

Remaining 12 patients were on teicoplanin and only one patient died within the $1^{\text {st }}$ week of positive blood culture (Table-1). $7^{\text {th }}$ day mortality following MRSA bacteremia was $24.5 \%$ and is significantly high compared to $7^{\text {th }}$ day mortality following SA bacteremia ( $0 \%$ mortality). Further, $7^{\text {th }}$ day mortality was significantly high ( $\mathrm{p}=0.03$, Fisher's exact test) among patients with vancomycin therapy compared to teicoplanin therapy (Table-1).

Since mortality was high in vancomycin-treated category, further analysis was done to assess contributory factors which influence the mortality. Following on or before $24 \mathrm{~h}$ of initiation of vancomycin treatment (Group 1), there were 37 survivors after day 7 and only 2 died as consequences of MRSA bacteremia. Following after $24 \mathrm{~h}$ in initiation of vancomycin treatment (Group 2), there were 2 survivors while 8 died as consequences of MRSA bacteremia (Table-2). When the factors individually considered, initiation of vancomycin treatment $>24$ h of blood culture positivity was significantly contributed to $7^{\text {th }}$ day mortality $\left(\chi^{2}=27.4 ; p=0.0002\right)$. The severity of MRSA infection (PITTS score; less severe: $<4$ and severe: $>4$ ) and having comorbidities (using $\mathrm{CC}$ index) was not significantly associated with $7^{\text {th }}$ day mortality (Table-2).

$7^{\text {th }}$ day mortality of MRSA bacteremia was further analyzed using logistic regression of similar parameters (comorbidities, severity of infection, and timely introduction of vancomycin). High PITTS score $(p<0.05)$ and initiation of vancomycin therapy after $24 \mathrm{~h}$ of receiving blood culture report $(\mathrm{p}<0.05)$ were independently affected the MRSA bacteremia patient's $7^{\text {th }}$ day mortality. Having comorbidities (CC index) did not show a significant impact on $7^{\text {th }}$ day mortality $(\mathrm{p}>0.05$; Table- 2$)$. The interactions between two independently significant factors (PITTS score and timely introduction of vancomycin treatment) were not significant.

\section{Discussion}

During the last 3 decades, the epidemiology and the nature of MRSA bacteremia have been analyzed in several studies [12-15]. However, the therapeutic outcome and the importance of timely introduction of vancomycin therapy need to be widely studied because that is lifesaving.

In our study, the percentage of MRSA bacteremia out of SA bacteremia was $9.3 \%$. Meantime, some of the European countries have shown a wide range of MRSA bacteremia proportions $(0 \%$ in Iceland to $52.4 \%$ in Malta) and a few countries such as France, the UK, and Slovenia have demonstrated a decline in MRSA bacteremia rates over recent years due to the implementation of multifaceted prevention programs [16]. Therefore, compared to other countries, THA, Sri Lanka shows a comparatively low prevalence of MRSA bacteremia.

According to our results, $4.5 \%$ had MRSA bacteremia out of the total cases of bacteremia. Hospitalacquired MRSA (HAMRSA) bacteremia was 3.03\%. In 2003, one of the studies conducted at Male Surgical Unit, National Hospital, Colombo, Sri Lanka; found that $6 \%$ prevalence of HAMRSA bacteremia [17]. This would be due the fact that surgical units are prone to acquire MRSA.

The highest number $(24.6 \%)$ of MRSA bacteremia was found in nephrology unit and that would be due to the large number of dialysis patients resulting from chronic kidney disease of unknown epidemic which prevails in the north central province. In 2005, dialysis patients in the USA accounted for $15.4 \%$ of all invasive MRSA infections [18]. Introduction of peritoneal dialysis catheter and arteriovenous line has significantly increased the likelihood of acquiring MRSA bacteremia. Although these procedures were

Table-1: Treatment outcome of vancomycin and teicoplanin regarding $7^{\text {th }}$ day mortality and survival rate.

\begin{tabular}{lccc}
\hline Anti-MRSA antibiotics & $\begin{array}{c}\text { Number treated } \\
\mathbf{n = 6 1}(\boldsymbol{\%})\end{array}$ & $\begin{array}{c}\text { Number of survivors after day } \mathbf{7} \\
\mathbf{n = 4 5 ( \% )}\end{array}$ & $\begin{array}{c}\text { Number of deaths before day } \mathbf{7} \\
\mathbf{n = 1 5}(\mathbf{\%})\end{array}$ \\
\hline Vancomycin & $49(80.3)$ & $39(75.5)$ & $10(20.4)$ \\
Teicoplanin & $12(19.7)$ & $12(24.5)$ & $1(8.3)$ \\
\hline
\end{tabular}

MRSA=Methicillin-resistant Staphylococcus aureus 
Table-2: Effect of timely initiation of vancomycin therapy, CC index, and PITTS score on $7^{\text {th }}$ day mortality and survival rates of patients with MRSA bacteremia.

\begin{tabular}{|c|c|c|c|c|}
\hline Parameter & $\begin{array}{c}\text { Number of survivors } \\
\text { and percentage }\end{array}$ & $\begin{array}{l}\text { Number of deaths } \\
\text { and percentage }\end{array}$ & $x^{2}$ value & p value \\
\hline \multicolumn{5}{|c|}{ Time from first blood culture to vancomycin } \\
\hline$<24$ h (Group 1) & $37(94.9)$ & $2(4.1)$ & 27.4 & $0.0002 *$ \\
\hline$>24 \mathrm{~h}$ (Group 2) & $2(20)$ & $8(80)$ & & \\
\hline \multicolumn{5}{|l|}{ PBS } \\
\hline$<4$ & $23(79.3)$ & $6(20.7)$ & 0.50 & 0.47 \\
\hline$>4$ & $16(75)$ & $4(25)$ & & \\
\hline \multicolumn{5}{|l|}{ CC index } \\
\hline Without comorbidities & $20(80)$ & $5(20)$ & 0.12 & 0.72 \\
\hline With comorbidities & $19(79.1)$ & $5(18.9)$ & & \\
\hline
\end{tabular}

$* p<0.05$ considered as significant. MRSA=Methicillin-resistant Staphylococcus aureus, $C C=$ Charlson comorbidity, PBS=Pitt Bacteraemia score

routinely done under sterile conditions, adherence to standard precautions, and assessment of sterility need to be done periodically [15].

In our study, the $7^{\text {th }}$ day mortality following SA bacteremia was zero and MRSA bacteremia was $13.1 \%$. A study conducted in Middle-East, Saudi Arabia, at a tertiary care hospital showed $36.4 \%$ mortality of the patients with MRSA bacteremia [19]. In South-East Asian countries, the mortality rate associated with serious SA infection was high as much as $50 \%$ [20]. The relative low $7^{\text {th }}$ day mortality in THA would be mainly due to early detection and implementation of appropriate therapeutic options such as antiMRSA antimicrobials and supportive therapy, on time.

The low $7^{\text {th }}$ day mortality of MRSA bacteremia was observed in the patients who received vancomycin treatment on or before $24 \mathrm{~h}$ of blood culture antibiotic susceptibility test report. This signifies the golden hour of initiation of vancomycin on MRSA bacteremia. The delay in introduction of vancomycin (starting after $24 \mathrm{~h}$ ) has significantly increased the mortality. When considering vancomycin susceptibility; all strains were sensitive to vancomycin. Still, the vancomycin-resistant and intermediate resistance SA is not documented and vancomycin use as main antiMRSA treatment modality in Sri Lanka.

The clinicians of THA seem reluctant to prescribe vancomycin as the first-line therapy to treat MRSA bacteremia because of vancomycin having a potential nephrotoxic effect, which further relates to the high prevalence of chronic kidney disease in north central province. In addition, having a significant delay of reaching the blood culture report from microbiology laboratory to ward settings would remarkably affects the $7^{\text {th }}$ day mortality rate. Even Sri Lanka comprises an advanced health-care setup, this scenario is quite common as other third world countries.

The highest mortality was observed in patients who were on vancomycin compared to teicoplanin. There are no reports in literature that allow us to contrast this effect. Further studies will be required to confirm the clinical relevance of this finding. Recent studies have shown suboptimal results in the treatment for bacteremia and endocarditis using vancomycin [21].
The reason for these findings has not been clarified yet. Other studies attribute this poorer effect of vancomycin to its use of treating the patients with more grave disease and worse prognosis [22].

On logistic model, the low PITTS score (mild infection) has significantly influenced the $7^{\text {th }}$ day survival of MRSA bacteremia patients. Further, Chi-squared test showed timely start of vancomycin treatment has significant influence on $7^{\text {th }}$ day mortality following MRSA bacteremia; however, the PITTS score did not influence significantly. The outcome variation between logistic regression and Chi-square test would be due to the effects of sample size. The CC index did not significantly influence the $7^{\text {th }}$ day mortality of MRSA bacteremia patients in either Chi-square of logistic regression model because of comorbidity would be mostly associated with $30^{\text {th }}$ day mortality.

\section{Conclusion}

We conclude that the start of vancomycin therapy within $24 \mathrm{~h}$ of receiving blood culture reports is significantly useful to reduce the mortality of MRSA bacteremia patients. In the future, conducting a largescale prospective study of timing, the vancomycin treatment on MRSA bacteremia will be important to elucidate the fact.

\section{Authors' Contributions}

JAASJ, MK, and WWK: Sample collection, bacterial cultivation, and biochemical confirmations. JAASJ: Clinical data collection and analysis. JAASJ, MK, WWK: Concept, study design, article writing, and revision and writing. All authors read and approved the final manuscript.

\section{Acknowledgments}

We acknowledge that T. L. Tennakoon manually entered data and involve database maintenance and L. D. Dissanayake traced record room statistics. This is a non-funded research.

\section{Competing Interests}

The authors declare that they have no competing interests. 


\section{References}

1. Abdulamir AS, Jassim SA, Hafidh RR, Bakar FA. The potential of bacteriophage cocktail in eliminating Methicillinresistant Staphylococcus aureus biofilms in terms of different extracellular matrices expressed by PIA, ciaA-D and FnBPA genes. Ann Clin Microbiol Antimicrob 2015;14:49.

2. McCarthy H, Rudkin JK, Black NS, Gallagher L, O’Neill E, O'Gara JP. Methicillin resistance and the biofilm phenotype in Staphylococcus aureus. Front Cell Infect Microbiol 2015;5:1.

3. Negi B, Kumar D, Kumbukgolla W, Jayaweera S, Ponnan P, Singh R, et al. Anti-methicillin resistant Staphylococcus aureus activity, synergism with oxacillin and molecular docking studies of metronidazole-triazole hybrids. Eur J Med Chem 2016;115:426-37.

4. Stefani S, Chung DR, Lindsay JA, Friedrich AW, Kearns AM, Westh H, et al. Meticillin-resistant Staphylococcus aureus (MRSA): Global epidemiology and harmonisation of typing methods. Int J Antimicrob Agents 2012;39:273-82.

5. Abramson MA, Bronstein MZ, Oden MA. Nosocomial methicillin-resistant and methicillin-susceptible Staphylococcus aureus primary bacteremia: At what cost? Infect Control Hosp Epidemiol 1999;20:408-41.

6. Wijesekara PN, Kumbukgolla WW, Jayaweera JA, Ravat DS. Review on usage of vancomycin in livestock and humans: Maintaining its efficacy, prevention of resistance and alternative therapy. Vet Sci 2017;4:6.

7. Anuradhapura Teaching Hospital. Available from: http:// www.health.gov.lk/eng/462. [Last cited on 2017 Feb 21; Last retrieved on $2017 \mathrm{Feb} 21]$.

8. Altun O, Almuhayawi M, Lüthje P, Taha R, Ullberg M, Özenci V. Controlled evaluation of the new BacT/Alert virtuo blood culture system for detection and time to detection of bacteria and yeasts. J Clin Microbiol 2016;54:1148-51.

9. Paterson DL, Ko WC, Von Gottberg A, Mohapatra S, Casellas JM, Goossens $\mathrm{H}$, et al. International prospective study of Klebsiella pneumoniae bacteremia: Implications of extended-spectrum beta-lactamase production in nosocomial infections. Ann Intern Med 2004;140:26-32.

10. Charlson ME, Pompei P, Ales KL, MacKenzie CR. A new method of classifying prognostic comorbidity in longitudinal studies: Development and validation. J Chronic Dis 1987;40:373-83.

11. SAS Institute Inc. SAS ${ }^{\circledR}$ 9.1.3. Language Reference: Concepts. $3^{\text {rd }}$ ed. Cary, NC, USA: SAS Institute Inc.; 2005.

12. Tong SY, Davis JS, Eichenberger E, Holland TH, Fowler VG Jr. Staphylococcus aureus Infections:
Epidemiology, pathophysiology, clinical manifestations, and management. Clin Microbiol Rev 2015;28:603-61.

13. Mejer N, Westh H, Schønheyder HC, Jensen AG, Larsen AR, Skov R, et al. Stable incidence and continued improvement in short term mortality of Staphylococcus aureus bacteraemia between 1995 and 2008. BMC Infect Dis 2012;12:260.

14. Groome MJ, Albrich WC, Wadula J, Khoosal M, Madhi SA. Community-onset Staphylococcus aureus bacteraemia in hospitalised African children: High incidence in HIVinfected children and high prevalence of multidrug resistance. Paediatr Int Child Health 2012;32:140-6.

15. Eshwara VK, Munim F, Tellapragada C, Kamath A, Varma M, Lewis LE, et al. Staphylococcus aureus bacteremia in an Indian tertiary care hospital: Observational study on clinical epidemiology, resistance characteristics, and carriage of the Panton-Valentine leukocidin gene. Int J Infect Dis 2013;17:e1051-5.

16. Lentino JR, Hennein H, Krause S, Pappas S, Fuller G, Schaaff D, et al. A comparison of pneumonia caused by gentamicin, methicillin-resistant and gentamicin, methicillin-sensitive Staphylococcus aureus: Epidemiologic and clinical studies. Infect Control 1985;6:267-72.

17. Corea E, de Silva T, Perera J. Methicillin-resistant Staphylococcus aureus: Prevalence, incidence and risk factors associated with colonization in Sri Lanka. J Hosp Infect 2003;55:145-8.

18. Morbidity and Mortality Weekly Report. Invasive Methicillin-Resistant Staphylococcus aureus Infections among Dialysis Patients - United States, Mortality Weekly Report 2005. Centers for Disease Control and Prevention (CDC), March 9; 2007. p. 197-9.

19. Nickerson EK, West TE, Day NP, Peacock SJ. Staphylococcus aureus disease and drug resistance in resource-limited countries in south and East Asia. Lancet Infect Dis 2009;9:130-5.

20. Madani TA, Al-Abdullah NA, Al-Sanousi AA, Ghabrah TM, Afandi SZ, Bajunid HA. Methicillin-resistant Staphylococcus aureus in two tertiary-care centers in Jeddah, Saudi Arabia. Infect Control Hosp Epidemiol 2001;22:211-6.

21. van Hal SJ, Fowler VG Jr. Is it time to replace vancomycin in the treatment of methicillin-resistant Staphylococcus aureus infections? Clin Infect Dis 2013;56:1779-88.

22. van Hal SJ, Jensen SO, Vaska VL, Espedido BA, Paterson DL, Gosbell IB. Predictors of mortality in Staphylococcus aureus Bacteremia. Clin Microbiol Rev 2012;25:362-86.

$* * * * * * * *$ 